\title{
Wenn gendered individuals einander begegnen
}

\section{Von der Notwendigkeit genderkompetenter Psychotherapie und Beratung}

\section{Brigitte Schigl}

Online publiziert: 17. Oktober 2016

(C) Der/die Autor(en) 2016. Dieser Artikel ist eine Open-Access-Publikation.

Zusammenfassung Ausgehend von den Überlegungen der gender theories zu Doing Gender und Dekonstruktion werden Implikationen für den therapeutischen Prozess beleuchtet. Aus einem gendersensiblen Blickwinkel betrachtet, zeigt sich schon die Geschichte der Psychotherapie auf engste mit Gender verwoben. Im individuellen psychotherapeutischen Prozess, in dem mit PatientIn und PsychotherapeutIn einander gleichermaßen gendered individuals begegnen, gibt es bestimmte Phasen und Themen, bei denen Gender eine besondere Rolle einnimmt. Hier emergieren im Doing Gender Dynamiken, die den Prozess färben und unhinterfragt auch risikoreich sein können Deshalb wird die Forderung nach Gendersensibilität und Genderkompetenz für alle PsychotherapeutInnen erhoben.

Schlüsselwörter Gender · Psychotherapie · Doing Gender · Genderkompetenz

\section{If gendered individuals meet}

Why we need gendersensibility in psychotherapy and counselling

\begin{abstract}
Based on the considerations of gender theories on doing gender and deconstruction, implications for the therapeutic process are illuminated. A gender sensitive perspective shows that the history of psychotherapy is closely interwoven with gender. In individual psychotherapeutic process, in which patient and psychotherapist encounter both as gendered individuals, there are certain stages and issues where gender plays a crucial role. In doing gender dynamics emerge that tone the process and can be risky if they are not aware and reflected. That is is why
\end{abstract}

Prof. Dr. B. Schigl, MSc ( $\varangle)$

Department für Psychotherapie und Biopsychosoziale Gesundheit, Donau Universität Krems, Krems, Österreich

E-Mail: brigitte.schigl@aon.at 
the demand for gender sensitivity and gender competence for all psychotherapists is being raised.

Keywords Gender · Psychotherapy · Doing gender · Gender-competence

\section{Die Geschichte: Gender und Psychotherapie - Wie alles begann}

Am Beginn der Entwicklung der Psychotherapie steht eine junge Frau, mit dem Pseudonym Anna O., die von einem Dr. Josef Breuer behandelt wird. Die vielfältigen Symptome der jungen Frau werden als ,hysterisch“ diagnostiziert. Hysterie war ein Krankheitsbild, dem der Psychiater Charcot, bei dem Breuers befreundeter Kollege, der junge Sigmund Freud lernte, zu publikumswirksamer Bekanntheit verholfen hatte (Holschbach 2002). Mit Freud bespricht Breuer den Behandlungsverlauf und experimentiert mit neuen Behandlungsmethoden. Anna O. ist die erste Patientin, die prototypisch in Freuds und Breuers Studien über Hysterie (1895) beschrieben wird. Die Entstehung der Psychoanalyse, Wurzel der tiefenpsychologischen und auch vieler humanistischer Psychotherapieverfahren, ist der Zusammenarbeit einer jungen Frau und ihres Arztes zu verdanken (Jones 1962, Bd. 1, S. 266). In deren Geschichte spiegeln sich soziale Veränderungen an der Wende vom 19. zum 20. Jahrhundert. Nach Jahrhunderten kaum hinterfragter patriarchaler Geschlechterordnung beginnt in dieser Zeit ein Vorwärtsdrängen junger Frauen aus bürgerlichen Kreisen in die Bildungseinrichtungen und Universitäten. In England fordern die Suffragetten der ersten Frauenbewegung das Erwerbs- und Wahlrecht und eine neue sittliche Ordnung der Gesellschaft. Das Leiden der Anna $\mathrm{O}^{1}$. und dessen Ausdruck sind nur vor diesem Hintergrund zu verstehen: Die Psychotherapie hat ihr Herkommen aus den individuell, intrapsychisch ausgedrückten Spannungen, die durch gesellschaftliche Bedingungen und Strukturen hervorgerufen waren (Nolte 2003). Die Behandlung derselben ist (zumindest anfangs) auf Augenhöhe: Der Arzt und die Patientin entwickeln gemeinsam eine ,talking cure“, die ihr hilft - nicht Medikamente oder Hypnose. Die Zugewandtheit einer anderen Person, die Entwicklung eines heilsamen Narrativs sind die wesentlichen Behandlungselemente. Etwas später begründet der junge Jakob Levi Moreno mit Freunden eine Gruppe von Prostituierten des Spittelsbergs, um deren Lebensbedingungen zu verbessern - ein erster Grundstein für die Entwicklung von Selbsthilfe und der Gruppenpsychotherapie (von Ameln et al. 2009, S. 193). Auch hier sollte gesellschaftlich hervorgerufenem Leid auf individueller Ebene begegnet werden. Die Entwicklung der humanistischen Psychotherapieverfahren geht mit der Kritik an den Bedingungen von Frausein in der Gesellschaft parallel (Schigl 2010): Nach der Vernichtung und dem Exodus der Psychotherapie während der nationalsozialistischen Diktatur in großen Teilen Europas kommt es in den späten 1970er und 1980er-Jahren zu einem Re-Import der meist in die USA exilierten und dort weiterentwickelten Therapieverfahren wie Psychodrama, Gestalttherapie oder der von Rogers dort begründeten Personzentrierten Psychotherapie. In

\footnotetext{
1 Anna O ist Berta Pappenheim, die später die deutsch-jüdische Frauenbewegung initiierte und sich als eine aktive Frauenrechtlerin im Kampf gegen Frauenhandel einen Namen machte (Brentzel 2002).
} 
diese Zeit fällt auch die zweite Frauenbewegung, deren Vertreterinnen oft aus den Frauengruppen der StudentInnenbewegung kommen. Diese Gruppen und sogenannte Consciousness raising groups setzen sich mit den Bedingungen von weiblichen Lebensvollzügen auseinander, thematisieren psychische Unterdrückung und Gewalt gegen Frauen, die Zurichtung der Leiblichkeit, die bestehende Ungleichbehandlung etc. In diesen Gruppen werden intensive Selbsterfahrungsprozesse angeregt und bearbeitet. Die zu Beginn noch ungeleiteten Gruppen (Kritik an ExpertInnen-Macht!) fördern die Beschäftigung mit den individuell-psychischen Reaktionen auf die gesellschaftlichen Strukturen. Die Kritik an der patriarchalen Kultur wird dabei direkt aus den Erfahrungen der Frauen abgeleitet - Das Private wird Politisch. Im Zuge dieses gemeinsamen Arbeitens eigneten sich Frauen Fähigkeiten zur Leitung solcher Gruppen an und absolvierten therapeutische Ausbildungen. Diese Therapeutinnen begannen auch die Prämissen und Methoden ihrer Therapierichtungen feministisch-kritisch zu hinterfragen; Analytikerinnen wie Nancy Chodorow, Christa Rohde-Dachser, Christiane Olivier, Jessica Benjamin oder die Therapeutinnen der deutschen feministischen Frauentherapiekongresse entwickelten aus der Kritik an den Theorien ihrer Therapieverfahren heraus ein neues frauengemäßes Verständnis für den Umgang mit dem psychischen Leiden. Die Therapeutinnen beziehen die gesellschaftlichen Umstände in ihr Verständnis für die Ätiologie bestimmter Störungsbilder mit ein und sehen individuelles Schicksal kollektiv mitverursacht. Dies soll auch in die Therapie miteinfließen im Wissen, dass das, was den Patientinnen widerfahren ist, Teil des Frauenlebens in unserer Gesellschaft ist und auch die Therapeutin selbst betrifft bzw. betreffen könnte (Schigl 2011).

Die öffentlich-politische Thematisierung dieser Inhalte beförderte die gesellschaftliche Diskussion um sexuellen Missbrauch, um Gewalt in familiären und PaarBeziehungen und ist als Diskurs um Trauma und Traumatisierung von allen Psychotherapieverfahren aufgegriffen worden. Diese Einbeziehung des gesellschaftlichen Hintergrunds und der Gender-Perspektive fand jedoch keinen breiten Widerhall in den Therapieverfahren selbst und blieb lange dem Engagement einiger weniger TherapeutInnen überlassen. Es gelang jedoch über Frauenberatungs- und Frauengesundheitszentren die Gedanken frauenspezifischer Angebote und Therapie außerhalb der therapeutischen Verfahren zu etablieren (Groth und Gallé 2010).

\section{Der Hintergrund: Hilfreiche Theorien aus den Gender-Diskursen}

Wenn wir uns Gender im psychotherapeutischen Prozess zuwenden, brauchen wir Analyseinstrumente, die uns helfen zu erkennen was sich hier manifestiert. Aus den Diskursen der Frauen- und Geschlechterforschung sind hier zwei Theorieansätze besonders fruchtbar für den Blick auf die Begegnungen von TherapeutInnen und PatientInnen.

\subsection{Doing Gender}

ist eine sozialkonstruktivistische Perspektive, die auf ethnologischen Forschungen beruht. 1987 formulierten Candance West und Don H. Zimmerman das Konzept 
des Doing Gender. Es besagt, dass all unsere Handlungen dadurch geprägt sind, ob wir weiblich oder männlich typisierend sozialisiert sind. Wenn sich zwei Individuen begegnen, ordnen sie einander automatisch nach der Genderzughörigkeit ein, sie erkennen einander als Frau oder Mann - und das ist das Wesentliche - sie verhalten sich jeweils danach. Mann mit Männern bringt subtil unterschiedlich gefärbte Interaktionen hervor als Frau mit Frauen und gemischtgeschlechtlichen Interaktionen sind von wieder anderen Dynamiken bestimmt. Carol Hageman-White (1993, S. 68) folgert daher, dass Gender ein performativer Akt, ein Tun vielmehr als ein Sein ist. Regina Gildemeister und Günther Robert postulieren ein Gefüge von inneren und äußeren Prozessen, die in Doing Gender zusammenwirken (2008). Gender ist sowohl Interaktion wie auch Institution, entlang derer die Welt in weiblich und männlich aufgeteilt wird - also als Prozess und Struktur zugleich. Beides verbindet sich in der individuellen Biografie, die in jedem Moment unsere Aktionen und Reaktionen mitbestimmt. Wichtig ist im Auge zu behalten, dass Gender dabei von weiteren sozialtypisierenden Variablen moderiert wird: Alter, Ethnie, sichtbare Zeichen religiöser oder kultureller Zugehörigkeit, körperliche Beeinträchtigungen u. a. sind ebenso wie Gender verhaltensbeeinflussend und können zusammenwirken, sich verstärken oder abschwächen, einzelne Kennzeichen zugunsten anderer in den Hintergrund treten lassen - ein „Doing difference“ (West und Fenstermaker 1995).

So wie in allen andern Interaktionen kommt Doing Gender auch im psychotherapeutischen Prozess zum Tragen. Es bestimmt, wie sich PatientInnen zu ihren TherapeutInnen in Abhängigkeit vom eignen und deren Geschlecht verhalten und umgekehrt. Dieser Gedanke ist für die Psychotherapie geradezu revolutionär, da in Überlegungen und Forschungsarbeiten zwar immer wieder das biologische Geschlecht, manchmal auch Gender der PatientInnen thematisiert wurde. Die Beteiligung der TherapeutInnen am wechselseitigen Vollzug der Interaktionen wurde allerdings in Bezug auf Gender außer Acht gelassen.

\subsection{Dekonstruktivistische Analyse}

Dieser Theoriebaustein aus den Gender-Diskursen wurde unter Bezugnahme auf verschiedene Philosophen von Judith Butler ausformuliert. Sie argumentiert, dass auch Doing Gender ein Diskurs um Geschlecht ist, der von Menschen mit ihrem Verständnis geschaffen wurde, also selbst Kulturprodukt. Die Unterscheidung von Sex und Gender, einem biologischem und einem sozial geformtem Geschlecht ist selbst Ausdruck einer gesellschaftlichen Praxis (Butler 1997). Denn man könnte ja annehmen, dass es einen ,natürlichen“ Geschlechtskörper jenseits der sozialen Konstruktionsprozesse gäbe - was nicht der Fall ist. Immer war menschliche Leiblichkeit sozial und kulturell überformt. Butler argumentiert, dass wir den Mechanismen dieser Konstruktion auf die Schliche kommen können, in dem wir gesellschaftliche Diskurse, also was, von wem, wann, wie gesagt wird - und v. a. auch was nicht gesagt wird, analysieren.

Dieser Blickwinkel hilft, in der Analyse der psychotherapeutischen Situation eine exzentrische Position einzunehmen, sich aus dem Spiel zu nehmen und zu reflektieren, was hinter dem, was inszeniert wurde, liegt. 


\section{Die Forschung - noch wenige Aussagen}

Geschlecht in die Forschung zu Gesundheit und Krankheit systematisch einzubeziehen ist ebenfalls eine Errungenschaft der 2. Frauenbewegung. Geschlechtsspezifische Gesundheitsförderung und Krankheitsrisiken (z. B. Kolip und Hurrelmann 2015) oder oft allein auf das weibliche Geschlecht bezogene Sichtweisen (z. B. aktuell Wimmer-Puchinger et al. 2016) sind in Medizin und auch Psychiatrie inzwischen State of the Art. Die Gender-Perspektive allerdings auch auf die BehandlerInnen auszudehnen, wird kaum getan.

Wenn es geschah oder geschieht, dann ging es in den quantitativ-empirischen Arbeiten oft darum zu sehen, ob weibliche oder männliche TherapeutInnen erfolgreicher in der Behandlung sind. Während in den 1980er und 1990er-Jahren diese Fragen eher zugunsten der weiblichen Therapeutinnen beantwortet wurden (Beutler et al. 1986), zeigen neuere Metaanalysen, dass es keinen klinisch relevanten Unterschied im Vergleich des Behandlungsoutcomes gibt (Bowman et al. 2001). Seitdem ist es recht ruhig um den Vergleich von männlichen und weiblichen TherapeutInnen geworden, Baldwin und Imel konstatieren im letzten Handbook of Psychotherapy and Behavior Change (2013, S. 259), dass es kaum neue Erkenntnisse zu den Unterschieden gibt, die das Geschlecht der TherapeutInnen macht bzw. machen könnte.

Viel interessanter sind da Publikationen von zumeist tiefenpsychologisch orientierten AutorInnen die das Übertragungs- und Gegenübertragungsgeschehen in den therapeutischen Prozessen beleuchten (z. B. Kerz-Rühling 1991). Sie machen damit den Sprung von der eindimensionalen Betrachtung von Sex als TherapeutInnen- oder PatientInnen-Variable zu einer interaktionalen Sicht eines gemeinsamen Prozesses, in dem die jeweiligen Genderzugehörigkeiten sich manifestieren und so jeweils typische Dynamiken entstehen - wobei eine Interpretation als „Übertragungsgeschehen“ aus Sicht des konstruktivistischen Paradigmas (vgl. 2) zu kurz greift. Denn es geht bei diesen Einfärbungen der Interaktionen nicht (nur) um individuelle Schemata und Relikte vergangener Beziehungen. Sie bilden vielmehr auch größere gesellschaftliche Wirklichkeiten $\mathrm{ab}$ - die eigene Biografie ist ja immer eingebettet in Kontext und Kontinuum (Petzold 2003).

In einer Studie an der Donau Universität zum Thema Risiken und Nebenwirkungen von Psychotherapie (Leitner et al. 2012) hat sich die Wichtigkeit einer solch interaktionalen Betrachtungsweise gezeigt: Während die bloße Analyse nach Frauen und Männern bei PatientInnen wie TherapeutInnen keine Unterschiede zeigten, fanden sich in der Berechnung der Daten für die jeweiligen möglichen 4 GeschlechtsKombinationen $^{2}$ signifikante Unterschiede, die als ein Beweis für Doing Gender in den jeweiligen Dyaden angesehen werden können (siehe 4.3).

\footnotetext{
${ }^{2}$ Eine Ausweitung der Kombinationen durch Miteinbezug von Inter*- Trans*- und Queeren Identitäten war aufgrund der klassisch zweigeschlechtlichen Quotierung der Studie nicht möglich.
} 


\section{Auf der Begegnungsbühne: Gender im therapeutischen Prozess ${ }^{3}$}

In der Psychotherapie begegnen einander Menschen, die aus ihrer jeweiligen social world ihre Version von Frau- oder Mannsein (er)leben. Aus den bisher skizzierten Grundlagen können wir folgern, dass BehandlerInnen ebenso wie ihre PatientInnen gendered individuals sind. Sie wachsen in spezifischen alltagskulturellen Kollektiven und Institutionen auf, die sie formen und sie reproduzieren diese mit jeder ihrer Interaktionen. Der entscheidende Gedankengang besteht nun darin, beide, TherapeutInnen und ihre PatientInnen in ihren Interaktionen miteinander in den Blick zu nehmen. PsychotherapeutInnen begegnen in gendertypisierender Art ihren PatientInnen (und vice versa) und müssen in gendersensibler Betrachtung gleichermaßen wie diese in den Blick genommen werden.

Wo und wie macht sich Doing Gender in den jeweiligen Kombinationen nun bemerkbar? Es sind dies bestimmte Situationen bzw. bestimmte Themen im Prozess, bei denen wir „die Konstrukteure von Gender auf frischer Tat ertappen können“ (Hagemann-White 1993). Sie stellen Kristallisationspunkte von Doing Gender dar und sind oft Anlass für gender troubles in den therapeutischen Beziehungen (genau in Schigl 2012, S. 105 ff.).

\subsection{Doing Gender im therapeutischen Prozess}

Das Handeln im therapeutischen Prozess ist ebenso wie alle anderen performativen Akte geprägt durch Gender und Gender wird in der Psychotherapie ebenso wie in anderen Interaktionen wechselweise hergestellt. Dies schafft für die InteraktionspartnerInnen Sicherheit und Ordnungsschemata, die zumeist nicht bewusst, aber hochwirksam sind; psychodramatisch könnte man sie als innere Skripts bezeichnen.

Schon von Beginn der Therapie an, vor dem Erstgespräch, entscheiden Phantasien und Annahmen von ÜberweiserInnen, ob für diesen oder diese PatientIn besser eine weibliche Therapeutin oder ein männlicher Therapeut geeignet wäre. Viele PatientInnen, die aus eigenem Entschluss und Antrieb in Psychotherapie kommen, überlegen schon vorher, ob es besser wäre bei einer Frau oder einem Mann die Behandlung durchzuführen, besonders weibliche Patientinnen machen sich darüber Gedanken (Schigl 1999) und bevorzugen eher gleichgeschlechtliche Dyaden.

In der ersten Sitzung kommt es beim ersten Sehen zu einem unweigerlichen gegenseitigen Einordnen des Gegenübers im Sinne von ,was für eine Frau, was für ein Mann ist dieser Mensch?“; diese unmittelbare Wirkung des ersten Eindrucks ist für beide AkteurInnen im therapeutischen Geschehen maßgeblich und Beobachtungen, wie sich PatientInnen in der Sitzung verhalten, wird von den TherapeutInnen oft auch diagnostisch genutzt. Natürlich wirken auch (Vor)urteile und Erfahrungen, die PatientInnen wie TherapeutInnen mit dem anderen Geschlecht gemacht haben, beeinflussen die Passung und bahnen Verhalten.

In besonders verunsichernden Momenten im therapeutischen Prozess - das ist meist dann wenn die therapeutische Beziehung thematisiert, oder die Therapie in Frage gestellt wird - können beide Parteien ebenfalls auf bekannte Gender-Schablo-

\footnotetext{
${ }^{3}$ Dies gilt für Psychotherapie ebenso wie für andere Beratungs- und Betreuungsprozesse.
} 
nen zurückgreifen; können hilfsbedürftig und nähesuchend oder auch wütend und fordernd werden und treffen auf ein Gegenüber, das ebenso entlang der Genderzugehörigkeit zu einer spezifischen Art des Umgangs neigt. Dies sind Phasen im Prozess, die sehr fehleranfällig sind, in denen das Risiko für problematische Entwicklungen erhöht ist (Schigl und Gahleitner 2013).

Es gibt Häufungen und Tendenzen in den Rückmeldungen der TherapeutInnen, wenn man sie zu typischen Resonanzen und Interaktionsmustern in den verschiedenen Geschlechterkonstellationen befragt (vgl. Schigl 2015). Generell wird sich bei PatientInnen des gleichen Geschlechts eher ein Gefühl von ,da kenne ich mich aus“, „da kann ich gut mitreden“ einstellen, als bei heterogenen Dyaden. Dieses gefühlte Gleich- bzw. Anderssein kann einerseits die Grundlage für genderspezifische Herangehensweise sein; sie kann andererseits auch zu Missverständnissen führen, wenn bei Gleichheit vorschnell Ähnlichkeiten und Wissen angenommen werden oder bei Andersheit zu vorsichtig und zögerlich interagiert wird.

\subsection{Doing Gender bei bestimmten Themen}

Es gibt bestimmte Themen in der Psychotherapie, die gendertypisierend bzw. -diskriminierend ,geladen“ sind; es sind dies Bereiche, in denen in unserem soziokulturellen Umfeld Frauen und Männer unterschiedliche Voraussetzungen haben, unterschiedlich reagieren bzw. die mit unterschiedlichen Empfindungen verknüpft werden. Oft sind es leibgebundene Thematiken, können wir doch Leiblichkeit ${ }^{4}$ als ein psycho-biologisch gegründetes, enkulturiertes Phänomen in einer persönlich einmaligen Form begreifen. Es sind dies Bereiche wie:

- Sexualität und Begehren und die damit verbundenen Emotionen; etwa unerfüllte Sehnsüchte, mangelndes Begehren, Impotenz oder sexuelle Dysfunktionen, Abweichungen von sexuellen Normvorstellungen, sexuelle Bedrängung beziehungsweise Grenzüberschreitungen (als TäterIn wie als BetroffeneR).

- Leiblichkeit und deren Bewertung, Schönheit und Attraktivität oder empfundener Mangel daran. Normvorstellungen von idealer Körperlichkeit (bei vielen jüngeren weiblichen Patientinnen sind diese Fragen zentral).

- Fragen der Identität, besonders in Bezug auf Gender: Welche Weiblichkeit oder Männlichkeit einE PatientIn entwickeln soll oder will; bei jüngeren PatientInnen die Konstituierung einer (erwachsenen) Geschlechtsidentität oder/und Auseinandersetzung mit der sexuellen Orientierung.

- Fruchtbarkeit und Reproduktion, z. B. die psychischen Implikationen von unerfülltem Kinderwunsch, gynäkologischen Problemen wie (schwierigen) Geburten oder von Schwangerschaftsunterbrechungen, Unfruchtbarkeit (als BetroffeneR bzw. PartnerIn).

- Psychische Implikationen von Erkrankungen der Geschlechtsorgane, wie Geschlechtskrankheiten, Brustkrebs, Gebärmutterhalskrebs oder Prostatakrebs mit den Folgen der jeweiligen medizinischen Therapien, sich leiblich neu zu verorten.

\footnotetext{
${ }^{4}$ Leiblichkeit kann als ein Zusammenwirken von bio-psycho und sozialen Prozessen verstanden werden, das Individuum ist ein Körper-Seele Geist-Subjekt im Kontext und Kontinuum (Petzold 2003).
} 
- Probleme in Paarbeziehungen: Vorstellungen von Beziehungsgestaltung, Machtverteilung in der Beziehung, Fragen der Treue, Verlassen-Werden und Trennung, (vergebliche) Werbung, psychische und physische Gewalt (als Opfer wie als TäterIn).

- Problemkonstellationen entlang der gesellschaftlichen Arbeitsteilung wie Kindererziehung, Hausarbeit, Pflege von Angehörigen, berufliche Karriereplanung, Teilzeit- oder Vollzeiterwerbsarbeit, Einheirat in Milieus mit von bisherigen Lebensentwürfen abweichenden Gendernormen.

- Soziale Probleme wie (mangelnde) Selbstbehauptung, Reaktanz, Konkurrenz, Macht und Aggression, Opfer oder TäterIn sein.

Wie immer wir in den Prozessphasen oder auf diese Themen reagieren, ist geprägt durch unsere Genderzugehörigkeit, die das „Klima“ und die Dynamiken in den therapeutischen Dyaden und Gruppen (wie auch in allen anderen sozialen Interaktionen) mitbestimmen. Doing Gender heißt sich entlang seiner Gendersozialisation $\mathrm{zu}$ verhalten und dieses Verhalten je nach Gender (und anderer sozialtypisierender Faktoren) des Gegenüber zu modifizieren, also Frausein bzw. Mannsein miteinander herzustellen. Dies hilft die unüberschaubar vielen Handlungsmöglichkeiten zu strukturieren und einzuschränken sowie gender accountability - zu beweisen. Diese „geschlechtliche Zurechnungsfähigkeit“ wird in Alltagssituationen und auch in der psychotherapeutischen Situation erwartet und hergestellt. PatientInnen erwarten von ihren männlichen und weiblichen TherapeutInnen subtil unterschiedliches Verhalten und vice versa. TherapeutInnen wie PatientInnen greifen auch in der therapeutischen Situation auf die ihnen in ihren social worlds vermittelten und individualbiografisch ausgeformten Weiblichkeiten und Männlichkeiten zurück.

\subsection{Doing Gender als Risiko}

Wenn wir im Therapieprozess bzw. bei den gender-typisierenden Themen nicht achtsam vorgehen, kann Doing Gender auch ein Risikofaktor im therapeutischen Prozess sein. Dies zeigt die Analyse der qualitativen und quantitativen Daten aus der RISK Studie der Donau Universität Krems (Schigl 2014): Bei der Analyse der anonymisierten Beschwerdedarstellungen, die im Beschwerdeausschuss des Psychotherapiebeirats im Zeitraum von 1991-2007 behandelt wurden, zeigten sich in etwa gleich viele Beschwerdefälle der Konstellation Therapeutin/Patientin (36\%) wie Therapeut/Patientin (38\%) Dies bedeutet einen deutlichen Überhang der Beschwerden von weiblichen Patientinnen über ihre männliche Therapeuten, da zum Zeitpunkt der Datenerhebung ca. doppelt so viele weibliche wie männliche TherapeutInnen tätig waren (Bestnet Informations-Service Gmbh 2016). In den Diskussionsgruppen von ExpertInnen aller Fachspezifika wurde zwar die Person des oder der PsychotherapeutIn als hauptsächlichster Risikofaktor genannt, allerdings Gender dabei nicht thematisiert. Offenbar ist das Risiko durch Doing Gender selbst den erfahrenen TherapeutInnen nicht bewusst.

Im quantitativen Teil der Online-Studie $(n=2056)$ zeigte sich im Faktor therapeutische Beziehung, dass es keine Mittelwertsunterschiede in der Beurteilung der therapeutischen Beziehungsqualität zwischen weiblichen und männlichen Pati- 
entInnen gab. Kombiniert man deren Angaben jedoch mit dem Geschlecht ihrer BehandlerInnen, so zeigt sich in der Konstellation weibliche Patientin und männlicher Psychotherapeut eine signifikant geringere Zufriedenheit mit der therapeutischen Beziehung. Aus den quantitativen Daten wurden auch drei mögliche Nebenwirkungen von Psychotherapie mittels Faktorenanalyse extrahiert: Sozioemotionale Labilisierung, Belastung und Isolation durch die Psychotherapie (vgl. Leitner et al. 2014, S. 20 f.). Auch hier schnitt die Dyade weibliche Patientin und männlicher Therapeut signifikant unterschiedlich ab. In dieser Konstellation wurde am häufigsten über unerwünschte Nebenwirkungen berichtet. Weiters wurde mittels eines Fragebogens katamnestisch erhoben, wie sich die Zufriedenheit (in verschiedenen Lebensbereichen) im Laufe der Psychotherapie verändert hatte. Die Zunahme an Lebenszufriedenheit im Rahmen der Therapie ist bei der Dyade weibliche Patientin und männlicher Therapeut insgesamt am geringsten. Bei jenen PatientInnen, die während der Therapie sogar eine Verschlechterung ihrer Lebenszufriedenheit erreichten, sind weibliche Patientinnen, die von männlichen Therapeuten behandelt wurden, signifikant häufiger vertreten. Die Daten der geschlechterhomogenen Dyaden zeigten tendenziell die besten Ergebnisse.

Bei derartigen Ergebnissen ist immer zu betonen, dass es sich hier um statistische Größen einer bestimmten Population (solche, die sich durch den Titel der Studie zu Risiken und Nebenwirkungen angesprochen fühlten, also wahrscheinlich eher unzufriedene PatientInnen) handelt. Natürlich können keine Rückschlüsse für einzelne konkrete Personen gezogen werden. Es bedeutet jedoch sehr wohl, dass wir eine nicht zu unterschätzende Einflussnahme der Gender-Dynamiken auf die Zufriedenheit mit der Psychotherapie haben. Dabei zeigen sich Effekte von Doing Gender man könnte interpretieren, dass in unseren Daten die Dyade von männlichem Therapeuten, der eine weibliche Patientin behandelt, für diese aufgrund ihrer GenderSozialisation nicht immer gesundheitsförderlich ist: Die patriarchale Dynamik eines wissenden Mannes, der der hilfesuchenden Frau beisteht, kann weibliche Erfahrungen von Abhängigkeit und Bedürftigkeit entlang eines Machtgefälles duplizieren und eher schwächend als stärkend wirken.

\section{Deshalb: Wir brauchen gendersensible und genderkompetente Psychotherapeutinnen und Psychotherapeuten!}

Diese Gender-Dynamiken können unbewusst ablaufen, eigenes Wissen und Handeln in Bezug auf Gender kann unreflektiert als Maß genommen werden. Die Perspektive Gender kann in schablonenhaften Zuschreibungen steckenbleiben, ohne die soziokulturelle Vielfalt unterschiedlicher Weiblichkeiten und Männlichkeiten mit in Betracht zu ziehen. Um dem gegenzusteuern müssen TherapeutInnen Gendersensibilität und Genderkompetenz entwickeln.

Zum Einstieg hilft es, sich über folgende Fragen Gedanken zu machen: Wie geschlechtsrollenkonform präsentiere ich mich als TherapeutIn meinen PatientInnen? Welche Weiblichkeiten/Männlichkeiten kenne und lebe ich, welche sind mir fremd? Wann nehme ich das Doing Gender meiner PatientInnen als von meinen eigenen Männlichkeits- und Weiblichkeitsvorstellungen ,,abweichend“ wahr? Wie bewerte 
ich das? Wann will ich es unterstützen, wann verunsichert es mich, will ich es korrigieren?

Damit TherapeutInnen Gender-Perspektiven entwickeln, ist zuallererst ein Wollen gefordert, sich mit diesen Thematiken überhaupt auseinanderzusetzen (Genderkompetenzzentrum 2003). Welchen Stellenwert will der oder die TherapeutIn Genderthemen einräumen? Wieweit hat sie oder er es als eine wichtige Dimension jedes Handelns erkannt? Ist das Wollen geweckt, kann Wissen für Gendersensibilität angereichert werden, sei es auf theoretisch-fachlicher, auf persönlich-selbsterfahrender oder supervisorisch-reflektierender Ebene (dazu Impulse Schigl 2012, S. 179 f.). Dies soll schließlich in ein Können, die eigentliche Genderkompetenz münden.

Gendersensible (auch geschlechterreflektierende) psychotherapeutische Praxis heißt im professionellen Handeln die Kategorie Geschlecht mitzudenken. Die genderspezifische Lebenspraxis von Frauen und Männern soll in Diagnose und Analyse miteinbezogen werden. Gendersensibilität nimmt beide Gender in ihren subkulturellen Besonderheiten (wie ihren Gemeinsamkeiten) wahr ${ }^{5}$.

Genderkompetenz in der therapeutischen Praxis meint die Umsetzung von Gendersensibilität im konkreten professionellen Handeln. Dies bedeutet nicht nur PatientInnen, sondern ebenso sich selbst als TherapeutIn als gendered individual in der Interaktion mit in den Blick zu nehmen, und daraus ergebende Dynamiken mit allen möglichen Geschlechtern zu berücksichtigen.

Folgende Aspekte können Gendersensibilität und Genderkompetenz illustrieren:

- Gendersensible Sprache verwenden.

- In den Therapieprozess die Analyse über weibliche und männliche Geschlechtertypisierungen und Lebensbedingungen hineinnehmen: Nicht nur die individuumzentrierte Sicht auf die Störung, das Leiden einbeziehen. Die individuellen Leidenszustände an gesellschaftliche Verhältnisse in den therapeutischen/ beraterischen Interpretationen rückzubinden ist Bestandteil genderkompetenter Praxis.

- Weitere sozialtypisierende Faktoren und Intersektionalitäten ${ }^{6}$ aus schicht-, kontext- und ethniespezifischen Bedingungen der Lebenswelt der PatientInnen berücksichtigen. Sensibilität gegenüber Gender in marginalisierten Gruppen wie z. B. MigrantInnen der ersten und zweiten Generation, homosexuellen Menschen, Frauen und Männern mit besonderen Bedürfnissen etc..

- Lernen am Modell: Der oder die TherapeutIn kann in gleichgeschlechtlichen Dyaden role-model für die PatientIn sein. (Die Resilienzforschung zeigt, dass wir

\footnotetext{
${ }^{5}$ Zum Unterschied von geschlechts- bzw. genderspezifischen Angeboten, die sich nur an ein Geschlecht richten. Sie gehen auf die Defizite oder Überkompensationen speziell für ein Gender in einer definierten Zielgruppe ein. Z.B. Frauenberatung, Männerberatung; Förderung von Selbstbewusstsein bei Mädchen, Beratungsangebote für alleinerziehende Väter, Gewaltprävention bei Burschen, Essstörungsprävention bei Mädchen etc.

${ }^{6}$ Intersektionelle Diskriminierung liegt vor, ,wenn - beeinflusst durch den Kontext und die Situation eine Person aufgrund verschiedener zusammenwirkender Persönlichkeitsmerkmale Opfer von Diskriminierung wird.“ (Becker Schmidt 2007, S. 57). Verschiedene Diskriminierungen können sich addieren und kummulieren - z. B. Obdachlos und alkoholkrank, Gehbehindert und Migrantin.
} 
am meisten von same-gender models profitieren, die gendertypisches Verhalten überschreiten vgl. Richter-Kornweitz 2011).

- Die Dynamiken im therapeutischen Prozess (4.1) sollen explizit in Bezug auf Gender analysiert werden. Doing Gender als Perspektive sollte bei eigenen und supervisorischen Überlegungen immer auch in Betracht gezogen und Gender so dekonstruiert werden.

- Besonders bei den Themen die Gender-Hotspots darstellen (siehe 4.2) sollte der Aspekt von Gender in der Interaktion immer mitgedacht, und idealiter auch angesprochen werden - ,Wie ist das für sie, dass Sie das jetzt mit einer Frau bzw. einem Mann besprechen?“

- Das Machtgefälle in der therapeutischen/beraterischen Arbeitsbeziehung soll gegebenenfalls angesprochen bzw. kann durch Solidaritätserfahrungen in der therapeutischen Beziehung nivelliert werden.

- Körperliche Berührung in der Therapie soll sensibel und bewusst gehandhabt werden.

In die Bearbeitung von seelischem Leid in der Psychotherapie hat vor allem durch die Bemühungen der Psychotherapeutinnen der zweiten Frauenbewegung die Beachtung der gesellschaftlichen Perspektiven Einzug gehalten. Individuelles Schicksal ist gesellschaftlich hervorgebracht - dies gilt für Frauen ebenso wie für Männer (und alle weiteren Gender) und muss immer (kritisch) mitbedacht werden.

Psychotherapie kann als tertiäre Sozialisation betrachtet werden, in der sich Bilder des Frau- bzw. Mannseins der PatientInnen (und vielleicht auch der TherapeutInnen) ändern können. Psychotherapie könnte der Ort sein, an dem eine gender-bezogene Identitäts- und Selbstwertentwicklung und Ausweitung der Handlungsoptionen im Doing Gender (und generell) befördert wird. Sie kann jedoch nur dann in dieser Weise wirksam werden, wenn unter den PsychotherapeutInnen das Wissen und die Bewusstheit um die jeweiligen persönlichen und gesellschaftlichen Bedingungen sowie die dahingehende Reflexion des eigenen Tuns im psychotherapeutischen Prozess Allgemeingut geworden sind. Ist dies nicht der Fall, können die besagten ,gender troubles“ auftauchen und Risiken für den Prozess bergen. Nötig ist deshalb seitens der TherapeutInnen die laufende Auseinandersetzung mit eigenen geschlechtstypisierenden Vorannahmen und dem eigenen Doing Gender in den therapeutischen Interaktionen. So kann die Gender-Färbung der Begegnung reflektiert, genutzt, abgepuffert und modifiziert werden - je nachdem was der therapeutische Prozess erfordert. Dies ist genderkompetente Psychotherapie.

Open access funding provided by Danube Universtity Krems University for Continuing Education.

Open Access Dieser Artikel wird unter der Creative Commons Namensnennung 4.0 International Lizenz (http://creativecommons.org/licenses/by/4.0/deed.de) veröffentlicht, welche die Nutzung, Vervielfältigung, Bearbeitung, Verbreitung und Wiedergabe in jeglichem Medium und Format erlaubt, sofern Sie den/die ursprünglichen Autor(en) und die Quelle ordnungsgemäß nennen, einen Link zur Creative Commons Lizenz beifügen und angeben, ob Änderungen vorgenommen wurden. 


\section{Literatur}

von Ameln, F., Gerstmann, R., \& Kramer, J. (2009). Psychodrama (2. Aufl.). Berlin: Springer.

Baldwin, S., \& Imel, Z.E. (2013). Therapist Effects: Findings and Methods. In M. J. Lambert (Hrsg.), Bergin and Garfield's Handbook of Psychotherapy and Behavior Change (6. Aufl. S. 258-297). Hoboken NJ: Wiley\& Sons.

Becker-Schmidt, R. (2007). Logiken der Differenzsetzung, Verschränkungen von Ungleichheitslagen und gesellschaftliche Strukturierung. In G.-A. Knapp \& A. Wetterer (Hrsg.), Achsen der Differenz. Gesellschaftstheorie und feministische Kritik (Bd. 2, S. 56-83). Münster: Westfälisches Dampfboot.

Bestnet Information-Service GmbH. (2016). Anzahl der TherapeutInnen nach Geschlecht. http://www. psyonline.at/contents/625/anzahl-der-therapeutinnen-nach-geschlecht-. Zugegriffen: 18. Juli 2016.

Beutler, L. E., Crago, M., \& Arizmendi, T.G. (1986). Therapist variables in Psychotherpeutic process and outcome. In A. E. Bergin \& S. E. Bergin (Hrsg.), Handbook of psychotherapy and behavior change (3. Aufl. S. 257-310). New York: Wiley \& Sons.

Bowman, D., Scogin, F., Floyd, M. R., \& Mc. Kendree-Smith, N. (2001). Psychotherapy length of stay and outcome: A meta-analysis of the effect of therapist sex. Psychotherapy, 38, 142-148.

Brentzel, M. (2002). Anna O. Berta Pappenheim. Biografie. Göttingen: Wallstein.

Butler, J. (1997). Körper von Gewicht. Die diskursiven Grenzen des Geschlechts. Berlin: Suhrkamp.

Freud, S., \& Breuer, J. (1895). Studien über Hysterie. Leipzig + Wien: Franz Deuticke. Neuauflage z. B. 1991 Frankfurt: Fischer Verlag.

Genderkompetenzzentrum (2003). Genderkompetenz. http://www.genderkompetenz.info/genderkompetenz2003-2010/gender/genderkompetenz.html. Zugegriffen: 18. Juli 2016.

Gildemeister, R., \& Robert, G. (2008). Geschlechterdifferenzierungen in lebenszeitlicher Perspektive. Interaktion - Institution - Biografie. Wiesbaden: VS.

Groth, S., \& Gallé, F. (2010). Frauengesundheit bewegt. In Frauen beraten Frauen (Hrsg.), In Anerkennung der Differenz. Feministische Beratung und Psychotherapie (S. 87-96). Gießen: Psychosozial.

Hagemann-White, C. (1993). Die Konstrukteure des Geschlechts auf frischer Tat ertappen? Methodische Konsequenzen aus einer theoretischen Einsicht. Feministische Studien. Kritik der Kategorie „Geschlecht" 11(2), 68-78.

Holschbach, S. (2002). Vom Bild der Leidenschaften zur Aufzeichnung der Symptome. Zu den zwei Visualisierungsparadigmen in Charcots ,,photographischer Klinik“. In T. Nusser \& E. Strowick (Hrsg.), Krankheit und Geschlecht. Diskursive Affären zwischen Literatur und Medizin (S. 123-142). Würzburg: Könighausen \& Neumann.

Jones, E. (1962). Das Leben und Werk von Sigmund Freud. Die Entwicklung zur Persönlichkeit und die großen Entdeckungen. 1856-1900. Bd. 1. Bern: Hans Huber.

Kerz-Rühling, I. (1991). Psychoanalyse und Weiblichkeit: Eine Studie zum Wandel psychoanalytischer Konzepte. Zeitschrift für psychoanalytische Theorie und Praxis, 6(3), 293-316.

Kolip, P., \& Hurrelmann, K. (Hrsg.). (2015). Handbuch Geschlecht und Gesundheit: Männer und Frauen im Vergleich. Bern: Hogrefe.

Leitner, A., Märtens, M., Höfner, C., Koschier, A., Gerlich, K., Hinterwallner, H., Liegl, G., Hintenberger, G., \& Schigl, B. (2012). Psychotherapie: Risiken, Nebenwirkungen und Schäden. Zur Förderung der Unbedenklichkeit von Psychotherapie. Endbericht. Krems, Österreich: Donau-Universität Krems. http://www.donau-uni.ac.at/imperia/md/content/department/psymed/forschungsartikel/ risk_bericht_11.09.2013.pdf. Zugegriffen: 17 Jul 2016.

Leitner, A., Schigl, B., \& Märtens, M. (Hrsg.). (2014). Wirkung, Risiken und Nebenwirkungen von Psychotherapie. Ein Beipackzettel für PatientInnen und TherapeutInnen. Wien: facultas.wuv.

Nolte, K. (2003). Gelebte Hysterie. Erfahrung, Eigensinn und psychiatrische Diskurse im Anstaltsalltag um 1900. Frankfurt: Campus.

Petzold, H. (2003). Die Integrative Identitätstheorie als Grundlage für eine entwicklungspsychologisch und sozialisationstheoretisch orientierte Psychotherapie. http://www.donau-uni.ac.at/imperia/md/content/ studium/umwelt_medizin/psymed/artikel/identitaetsth.pdf. Zugegriffen: 17 Jul 2016.

Richter-Kornweitz, A. (2011). Gleichheit und Differenz - Die Relation zwischen Resilienz, Geschlecht und Gesundheit. In Z. Margherita (Hrsg.), Handbuch Resilienzförderung (S. 240-274). Wiesbaden: VS.

Schigl, B. (1999). Wirkungen und Wirkfaktoren von Gestalttherapie aus katamnestischer Sicht der KlientInnen - Ausgewählte Ergebnisse einer evaluativen Studie. In R. Hutterer-Krisch, I. Luif \& G. Baumgartner (Hrsg.), Neue Entwicklungen in der Integrativen Gestalttherapie. Wiener Beiträge zum Theorie-Praxis Bezug (S. 222-252). Wien: facultas. 
Schigl, B. (2010). Feministische und Gendertheorie-Diskurse und ihre Bedeutung für das psychosoziale Feld. Journal für Psychologie 18/3. http://www.journal-fuer-psychologie.de/index.php/jfp/article/ view/32/180. Zugegriffen: 17 Jul 2016.

Schigl, B. (2011). Frauenspezifische Psychotherapie. In G. Stumm (Hrsg.), Wege zur Psychotherapie (S. 428-431). Wien: Falter.

Schigl, B. (2012). Psychotherapie und Gender. Konzepte. Forschung. Praxis. Welche Rolle spielt die Geschlechtszugehörigkeit im therapeutischen Prozess? Wiesbaden: Springer VS.

Schigl, B. (2014). Gendered Risk: Risikofaktor Geschlecht? In A. Leitner, B. Schigl \& M. Märtens (Hrsg.), Wirkung, Risiken und Nebenwirkungen von Psychotherapie. Ein Beipackzettel für PatientInnen und TherapeutInnen (S. 51-65). Wien: facultas.wuv.

Schigl, B. (2015). Doing Gender im psychotherapeutischen Prozess - braucht Dekonstruktion. Psychosozial, 2015(2), 39-54.

Schigl, B., \& Gahleitner, S. (2013). Fehler machen - aus Fehlern lernen? - Perspektiven zur Klassifizierung von psychotherapeutischen Fehlern und dem Umgang damit. In Psychotherapiewissenschaft. Jg. 3/1. http://www.psychotherapie-wissenschaft.info/index.php/psy-wis/article/view/1001. Zugegriffen: 18 Jul 2016.

West, C., \& Zimmerman, D. H. (1987). Doing Gender. Gender \& Society. 1(2), 125-151.

West, C., \& Fenstermaker, S. (1995). Doing Difference. Gender \& Society. 9(1), 8-34.

Wimmer-Puchinger, B., Gutiérrez-Lobos, K., \& Riecher-Rössler, A. (Hrsg.). (2016). Irrsinnig weiblich Psychische Krisen im Frauenleben. Hilfestellungen für die Praxis. Berlin: Springer.

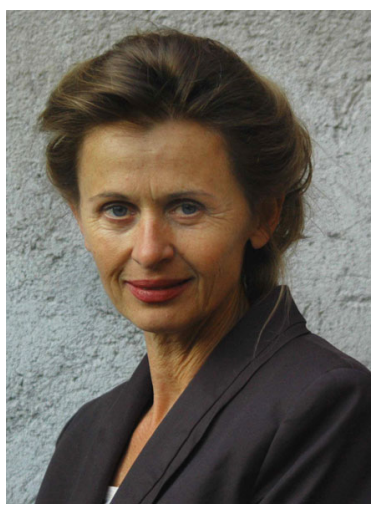

Prof. Dr. Brigitte Schigl, MSc Klinische und Gesundheitspsychologin, Psychotherapeutin, Supervisorin. Arbeitet in freier Praxis in Wien und Krems. Lehrende am Department für Psychotherapie und Biopsychosoziale Gesundheit der Donau Universität Krems (Integrative Therapie, Supervision \& Coaching). Psychotherapie- und Supervisions-Forschung (Therapeutenvariable, Gender) 